\title{
Optimal Buyer-Seller Inventory Models in Supply Chain
}

\author{
Gaobo Li \\ Shandong Women's University, Jinan, 250300,China \\ email: ligaobo_1979@163.com
}

Keywords: Inventory; Deterioration merchandise; Joint cost; Quantity discount

\begin{abstract}
In this study, we focus improvement inventory models on two member's simple buyer-seller system in supply chain. An inventory model is developed for a time-varying demand deteriorating inventory to determine the optimal order interval and discount price so that the joint total cost is minimized during a finite planning horizon.
\end{abstract}

\section{Introduction}

The increasing of globalization of markets and competition pressure, forces companies to develop the partnership of supply chain to respond quickly to customer requirement. Supply chain management is the use of the effective integration of suppliers, manufactures, distributors, distribution and inventory and so on, so that goods can be in the right quantity and be sent to the right place at the right time, making the system(system-wide)cost to a minimum and meeting the required service level at the same time.

In the supply chain, inventory of goods is the main operating activity and the source of profit. In order to meet the operating activity's needs and give the goods to the customer on time, both buyers are appropriate to hold goods. However, with operating activity for both buyers, as well as the storage and management of goods, the relevant inventory cost is brought for both buyers. The planning and management performance of the inventory are vital to the survival and development of the company. Both buyers in supply chain are committed to make the relevant inventory cost to a minimum and the profit to the highest.

In order to make inventory model agree with the current of the operating activity ,integrated inventory model considering multiple factors gradually increases, which makes the derivation of the model more complex, so that part of literature discussing the model constructed by the inventory problem only derives approximate solution of the inventory model. That shows there is room for improvement of storage of the inventory problem. Therefore, this research based on these factors and Gao yushu's(1996) hypothesis, uses different mathematical derived systems and constructs optimal buyer-seller inventory model of supply chain, to explore the moderate simplification and possibilities of improvement of the relevant inventory model and derive the optimal decision-making solution of the inventory model.

\section{Study on the theories of the inventory model}

\section{Study on the theories of the buyer-seller inventory model}

Douglas(1996) put forward a lot of traditional inventory models focused on the decision of buyer's optimal order number, and such models may ignore the two chances: Firstly, the investment of material handling equipment or data exchange technology, for example, electronic information exchange system, can reduce the cost without changing the ordering strategy. Secondly, the company can find the optimal joint order number for both buyers and decide how to allocate the saving value through the parties' consultation. Lal and Staelin(1994) proposed the response model for the seller of the buyer's pricing plans, to encourage the buyer to order even greater number of movements per time and make both buyers relevant inventory cost minimum. Nahan(1994) put forward the optimal quantity discount price for the seller of the buyer's order level ,to make the seller obtain the maximum profit and not increase the seller's cost of the inventory model. In the 
random condition, Banerjee(1996) processed the analysis model controlled by the single good inventory for the development of the single seller and the multi-buyer and used the EDI to make the coordination in this model become more easy. In the retail industry,Liu Yuchang(1999) investigated the decision-making model of the inventory of the price in suppliers and retailers and the order quantity when the buyer's demand is influenced by the price.

Most of them just contrast the optimal model and develop the model of calculus in the buy's opinion, and not analyze how to develop the best quotation architecture of the seller to influence the buyer's purchasing strategy. To investigate the inventory for the seller, the buyer, or both buyers is to make the seller and the buyer's relevant inventory cost and both buyers' joint cost minimum. However, if only the buyer or the seller profits, the system will not last long. Many studies have shown that, both buyers can reduce the joint cost and increase the profit in the coordination and cooperation mode. So this research is mainly to explore the optimal buyer-seller inventory model.

\section{Study on the relevant theories of the deterioration inventory model}

Ghare and Scrader(1973) considered the problem of the deterioration merchandise inventory in their development inventory model. Misra(1975) proposed the inventory model whose replenishment rate is finite, and the deterioration of the merchandise obeys the exponential distribution and Webb distribution with two parameters. In accordance with the buyer and the seller in single, Gao Yushu(1996) explored the optimal inventory model in the fixed index loss when the seller's replenishment rate is finite and infinite, and the buyer's demand changes over time and is liner. Considering the demand is fixed and the index loss is fixed, Wu Meiying(1999)put the concept of the small batch transportation into the inventory to obtain the optimal joint cost of the multi-buyer and the single seller. In the light of the perishable goods, Han Jiahong(2002)built up the multi-stage order strategy in period of validity to solve the problem of single stage order in period of validity of the traditional inventory model.

\section{Study on the theories of the quantity discount inventory model}

In the commercial activities, the buyer should consider how much the order quantity is, when it is time to replenish the inventory and how much to minimize the total relevant cost. But the seller always tries to provide the quantity discount to influence the buyer's order behavior and encourage the buyer to order more, looking for reducing the setup cost and the transaction cost.

Susan and Zhimin(1995) identified three main areas of research: The first is to explore the inventory model in the point of the buyer's optimal order quantity ,or the buyer obtains the quantity discount of the seller because of a large order. The second is to explore the inventory model in the point of the seller, and to achieve the purpose of the lowest transaction cost through quantity discount or difference in price. The third is to explore the joint inventory model in the point of both buyers and to improve transaction efficiency taking into the interests of both buyers.

Lin Xiuyi(1997)used the equilibrium theory to explore the optimal system order quantity ,order price and delivery times for both buyers under the different subordinate relationship ,and to establish a price discount model in multiple distribution environment to analyze the sensitivity of the changes of the demand, the budget of the buyer, the order cost, the shortage percentage, the distribution cost and the holing cost.

\section{Study on the theories of the profit sharing}

Factors of estrangement between buyers and sellers are: inconsistency, critical incident, conflict and perceived differences. There are many potential sources of conflict between buyers and sellers, including: price, quality, delivery, delayed payment, financial penalties for non-performance, order cancellations and intellectual property. But all of the procurement professionals are very clear most time should be spent on the problem about the purchase price. The coordination and cooperation of both buyer is advantageous to reduce the conflict. How to distribute the co-creation profit of 
coordination and cooperation is a key factor to make both buyers sustain cooperation or increase conflict. Therefore, the problem of distribution of profits in the optimal buyer-seller inventory model is also valued by researchers.

\section{Construction of the inventory model}

\section{Research the framework}

According to the theory above, a lot of researches improve the transaction efficiency to make the buyer-seller relevant inventory cost minimum. Study the theories of the deterioration inventory is to explore the optimal inventory model under different circumstances of the loss. Study the theories of the quantity discount is to seek to derive the optimal price and replenishment strategy in various quantity discount model to minimize the buyer-seller relevant inventory cost. Study on the theories of the profit sharing is to construct the model of profit sharing as the additional profit sharing system for both buyers considering the weight factors or using different negotiation model after obtaining the minimum joint cost. We find in the study that Gao Yushu(1996)explored the optimal inventory model of the deterioration merchandise when the buyer's demand changes over time. In order to make the buyer-seller joint cost minimum, he used trial and error method to determine the optimal order cycle and the optimal approximate solution to the discount price. This study based on the assumption by Gao YUshu(1996) uses different analytical methods to derive the optimal order cycle and the optimal solution to the discount price to minimize the buyer-seller joint cost.

According to to the discussion above considered, we put forward the research framework, as shown on fig.1.
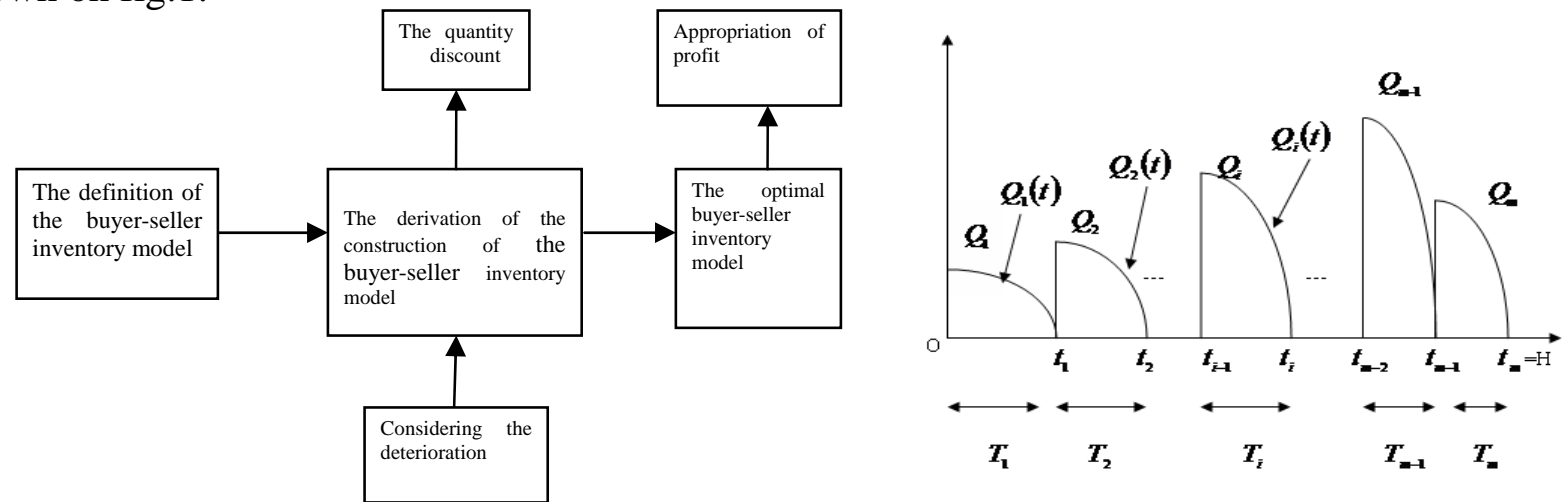

Fig.1. The research framework of the thesis

Fig.2. The model that the demand changes with time,

relationship between inventory levels and time

\section{The basic assumption of the inventory}

The symbols used in the inventory are as follows:

$H$ :Planning period; $m$ : The order number in the planning period; $P$ :The original price of the goods; $p i$ :The discount price of the goods in the i cycle, $i=1,2, \ldots, m ; \theta$ : The deterioration rate of the goods; $r$ :The inventory ratio in unit time of the buyer's per unit; $K$ :The order processing cost of the seller; $S$ :The setup cost of the seller; $A$ : The order processing cost of the buyer; $B S$ :The saving value of the buyer in per unit time; $S S$ : The saving value of the seller in per unit time; $\alpha$;The distribution proportion of the saving value in buyer-seller system; $I i$ :The total capacity of the inventory in the $i$ cycle; $T i$ :Any order cycle, $i=1,2, \ldots, m$; $i$ :The time point of the inventory depletion in any cycle, $i=1,2, \ldots, m \cdot t_{0}=0, t_{m}=H ; T=t_{i}-t_{i-1}, i=1,2 . . . m-1 ; t_{i}=t_{i-1}+T_{i}, i=1,2 . . . m-1$ $t_{i}=t_{i-1}+T_{i}, i=1,2, \ldots m-1, T_{0}=Q T_{m}=H-t_{n+1} D(t)$ :The demand rate, a function of time, $\left.D t\right)=a+b t 0 \leq t \leq H ; a$ 
and $b$ are constants, $a \geq 0, a+b t \geq 0$; When $b \succ 0$, the demand increases with time; When $b \prec 0$, the demand decreases with time.

$B\left(p_{i}, T_{i}\right)$ : The cost of the buyer in the $i$ cycle, $i=1,2, \ldots, m$.

$S\left(p_{i}, T_{i}\right)$ : The cost of the seller in the $i$ cycle, $i=1,2, \ldots, m$.

$J\left(p_{i}, T_{i}\right)$ : The joint cost of both buyers in the $i$ cycle, $i=1,2, \ldots, m$.

$Q(t)$ : The inventory cost in any time $t$ in the $i$ cycle.

\section{To solve the inventory model}

This chapter will derive the optimal inventory model and use the analysis method to obtain the solution to the optimal buyer-seller inventory model. According to the hypothesis, when the demand increases linearly with time, relationship between inventory levels and time is as shown in fig.2.

If $Q_{i}(t)$ denotes the $i$ cycle, the inventory level at any time $t$, and $t_{i-1} \leq t \leq t_{i}$. The inventorylevel can be expressed by the following equation:

$$
\frac{d}{d t} Q_{i}(t)+\theta Q_{i}(t)+(a+b t)=0 \text {, Namely, } Q_{i}(t)=\frac{b-a \theta-b \theta t}{\theta^{2}}+e^{-\theta t} \cdot c
$$

Fig. 2 shows that, $Q_{i}\left(t_{i}\right)=0$, let $t=t_{i}$ and put it into(1)

So, $Q_{i}(t)=e^{-\theta t}\left(\frac{a \theta+b \theta t_{i}-b}{\theta^{2}} e^{\theta t_{i}}\right)-\left(\frac{a \theta+b \theta t-b}{\theta^{2}}\right)$

Let $t=t_{i-1}$, the initial inventory level $Q_{i}$ in the $i$ cycle is given by:

$$
Q_{i}=Q_{i}\left(t_{i-1}\right)=e^{-\theta t_{i-1}}\left[\frac{e^{\theta t_{i}}\left(a \theta+b \theta t_{i}-b\right)}{\theta^{2}}\right]-\left(\frac{a \theta+b \theta t_{i-1}}{\theta^{2}}\right)
$$

The total inventory $I_{i}(t)$ in the $i$ cycle is given by:

$$
I_{i}(t)=\int_{t_{i-1}}^{t_{i}} Q_{i}(t) d t=\int_{t_{i-1}}^{t_{i}}\left(\frac{a \theta+b \theta t_{i}-b}{\theta^{2}} e^{\theta t_{i}} \cdot e^{-\theta t}+\frac{b-a \theta-b \theta t}{\theta^{2}}\right) d t
$$

Because $Q_{i}\left(t_{i}\right)=0, i=1,2, \ldots m$ and $T_{i}=t_{i}-t_{i-1}$, so (4)is given by:

$$
I_{i}(t)=\int_{t_{i-1}}^{t_{i-1}+T_{i}}\left(\frac{a \theta+b \theta t_{i}-b}{\theta^{2}} \cdot e^{\theta t_{i}} \cdot e^{-\theta t}+\frac{b-a \theta-b \theta t}{\theta^{2}}\right) d t
$$

When $i=1, t_{1}=T_{1}=T$ (for the sake of convenience, here $T_{1}=T$ ) and put $t_{0}=0$ into(5),you can obtain the inventory in the first cycle:

$$
I_{1}=\left(\frac{a \theta+b \theta T-b}{\theta^{3}}\right)\left(e^{\theta T}-1\right)-\left(\frac{a \theta T+\frac{1}{2} b \theta T^{2}}{\theta^{2}}\right)
$$

The buyer's demand in the cycle is:

$$
D_{i}=\int_{t_{i-1}}^{t_{i}}(a+b t) d t=a t_{i}+\frac{1}{2} b t_{i}^{2}-a t_{i-1}-\frac{1}{2} b t_{i-1}^{2}
$$


Let $i=1, t_{i}=t_{1}=T_{1}=T$

The relevant cost for both buyers in the first cycle per unit time can be given by:

The relevant cost for the buyer in the first cycle per unit time can be given by:

$$
\frac{B\left(p_{1}, T\right)}{T}=\frac{A}{T}+\frac{p_{1} Q_{1}}{T}+\frac{p_{1} r I_{1}}{T}
$$

The relevant cost for the seller in the first cycle per unit time can be given by:

$$
\frac{S\left(p_{1}, T\right)}{T}=\frac{S+K}{T}+\frac{\left(P-p_{1}\right) Q_{1}}{T}
$$

The relevant joint cost for both buyers in the first cycle per unit time can be given by:

$$
\frac{J\left(p_{1}, T\right)}{T}=\frac{A+S+K}{T}+\frac{P Q_{1}}{T}+\frac{p_{1} r I_{1}}{T}
$$

The minimum cost proves to be existed (the situation that the buyer and the seller is not united):

Let $\frac{B(P, T)}{T}=B(T)$, minimize the relevant cost for the buyer in the first cycle per unit time, the necessary condition is: $B^{\prime}(T)=0$. Put $I_{1}, Q_{1}, p_{1}=P, i=1$ into(8), $B(T)=\frac{A}{T}+P\left[e^{\theta T}\left(\frac{a}{\theta T}+\frac{b}{\theta}-\frac{b}{\theta^{2} T}\right)-\frac{a}{\theta T}+\frac{b}{\theta^{2} T}\right]+\operatorname{Pr}\left[\left(\frac{a}{\theta^{2} T}+\frac{b}{\theta^{2}}-\frac{b}{\theta^{3} T}\right)\left(e^{\theta T}-1\right)\right]-\left(\frac{a}{\theta}+\frac{b T}{2 \theta}-\frac{b}{\theta^{2}}\right)$

Seek the derivative and second derivative of (11), $\because b \theta^{2} T\left(e^{\theta T}-1\right) \succ 0 \quad \therefore B^{\prime \prime}(T) \succ 0 \quad \therefore B^{\prime}(T)$ is a increasing function.Also, $B^{\prime}(0)=-\frac{A}{T^{2}} \prec 0, B^{\prime}(\infty)=\lim _{T \rightarrow \infty}(B(T))=+\infty$ So $T^{*}$ exists, such that $B^{\prime \prime}(T) \prec 0$, when $T \in\left(0, T^{*}\right) ; B^{\prime \prime}(T) \succ 0$, when $T \succ T^{*}$. The graph of function $B^{\prime \prime}(T)$ is shown as fig .3.

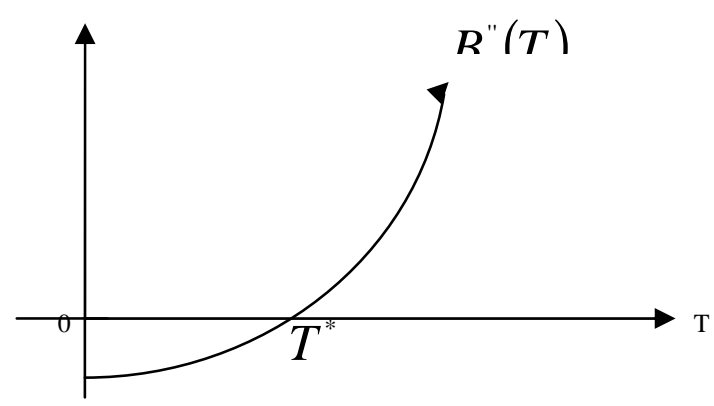

Fig .3. The graph of function $B^{\prime \prime}(T)$

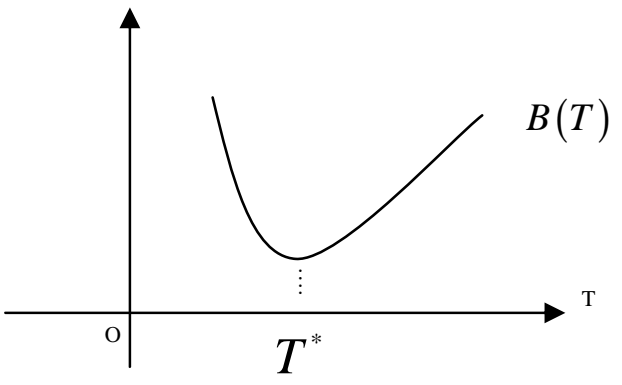

Fig.4.The graph of function $B(T)$

It is known that $B^{\prime}\left(T^{*}\right)=0$, also $B^{\prime}(T) \prec 0, T \in\left(0, T^{*}\right), \quad B(T)$ decreases; $B^{\prime}(T) \succ 0, T \succ T^{*}$, $B(T)$ increases. The graph of function $B(T)$ is shown as fig .4.

So, the minimum cost $B(T)$ proves to be existed in $T=T^{*}$, namely the minimum cost is $B\left(T^{*}\right)$.

The minimum buyer-seller joint cost $J(T)$ can be proved to be existed in $T=T^{* *}$ in a similar way, namely the minimum cost is $J\left(T^{* *}\right)$. Here is no proof. 


\section{Study on the optimal inventory model}

Based on the analysis of the derivation of the model, the relevant cost for both buyers may change as follows:

When the seller doesn't provide the quantity discount in the first cycle, the buyer has to seek the optimal order cycle $T^{*}$ to minimize the relevant cost per unit time. The relevant cost for both buyers is respectively:

$\frac{B\left(P, T^{*}\right)}{T}$ ( The relevant cost in the smallest unit of time of the buyer in the order cycle $T^{*}$ )

$\frac{S\left(P, T^{*}\right)}{T^{*}}$ ( The relevant cost in the smallest unit of time of the seller in the order cycle $T^{*}$ )

The minimum joint cost for both buyers is obtained in the order cycle $T^{* *}$. The relevant cost for both buyers is respectively:

$\frac{B\left(p_{1}, T^{*}\right)}{T \cdot{ }^{*}}$ ( The relevant cost per unit time of the buyer in the order cycle $T^{* *}$ )

$\frac{S\left(p_{1}, T^{* *}\right)}{T^{* *}}$ ( The relevant cost per unit time of the buyer in the order cycle $T^{* *}$ )

$T^{* *} \succ T^{*}$, so if the seller doesn't provide the quantity discount in the situation that the buyer and the seller unite, namely $p_{i}=P$, the relevant cost of the buyer per unit time is: $\frac{B\left(P, T^{*}\right)}{\left.T{ }^{*}\right)}>\frac{B\left(P, T^{*}\right)}{T^{*}}$, which means the relevant cost increases in the situation that the buyer unites and the intention of the buyer is influenced. If the seller provides the quantity discount to encourage the buyer in the situation that the buyer and the seller unite, the relevant cost of the seller per unit time is: $\frac{S\left(p_{1}, T^{*}\right)}{\left.T \cdot{ }^{*}\right)}>\frac{S\left(P, T^{*}\right)}{T}$, which means the relevant cost increases in the situation that the seller provides quantity discount and the joint intention of the seller is also influenced.

Based on the analysis above, in order to make the buyer and the seller unite and minimize the joint cost for both buyers, we must seek the best discount price $p_{1}^{*}$ that replaces $p_{1}$ and the optimal order cycle $T^{* * *}$ that replaces $T^{* *}$, meeting the condition that $\frac{B\left(p_{1}^{*}, T^{* * *}\right)}{T^{* * *}} \leq \frac{B\left(P, T^{*}\right)}{T^{*}}$ and $\frac{s\left(p_{1}^{*}, T^{* *}\right)}{T^{* *}} \leq \frac{S\left(P, T^{*}\right)}{T^{*}} \cdot p_{1}^{*}$ and $T^{* * *}$ are proved to be existed , and this paper will not demonstrate in detail.

\section{Conclusion}

Through the analysis of inventory model via the derivation, under the situation that the buyer and the seller unite, and according to the model this research provides, we get the optimal order cycle and the optimal quantity discount to achieve the win-win goal of minimizing the joint cost and saving the relevant cost for both buyers.

\section{References}

[1].Gao Yushu, The buyer-seller inventory model of the deterioration merchandise with time-varying demand, industrial engineering, 1996.

[2]. Mines, Creating World Class Suppliers[M], Pitman Publishing,Landon,1994,(10).

[3].Wang Yingyun, Supply chain management-the method of modeling and data mining[M],Beijing: Tsinghua University press,2005,(5).

[4].Lu Juqiang, The enterprise logistics outsourcing and research of partner selection[J],Journal of management science,2006. 